\title{
Captures
}

Figures, théories et pratiques de l'imaginaire

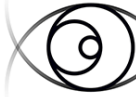

\section{Identité liminaire dans 4.48 Psychose de Sarah Kane Une lecture queer}

C A P T U R E S Figures, théories et pratiques de limaginaire revue interdisciplinaire

\section{Aramesh Yaghoubi}

Volume 3, Number 1, May 2018

La notion d'«autochtonie»

URI: https://id.erudit.org/iderudit/1055841ar

DOI: https://doi.org/10.7202/1055841ar

See table of contents

Publisher(s)

Figura, Centre de recherche sur le texte et l'imaginaire

ISSN

2371-1930 (digital)

Explore this journal

Cite this article

Yaghoubi, A. (2018). Identité liminaire dans 4.48 Psychose de Sarah Kane : une lecture queer. Captures, 3(1). https://doi.org/10.7202/1055841ar
Article abstract

This study of Sarah Kane's play 4.48 Psychosis puts forth in a queer perspective. The play sees the emergence, on stage and in text, of what Judith Butler refers to as "queer identity". Its text invents a language of ambivalence between voice and writing, between the masculine and the feminine: a language that transgresses binary categories. The body of the protagonist settles in a mental and symbolic space where several voices intertwine and hybridize; this space is ambivalent, between life and death, between the sublime and the obscene. The phase of liminality in which the piece unfolds becomes a space-time conducive to the re-appropriation of the body by a writing that replaces the flesh.
Tous droits réservés (c) Aramesh Yaghoubi, 2018

\section{(c) (i) $\Theta$}

This document is protected by copyright law. Use of the services of Érudit (including reproduction) is subject to its terms and conditions, which can be viewed online.

https://apropos.erudit.org/en/users/policy-on-use/ 


\section{Identité liminaire dans 4.48 Psychose de Sarah Kane}

\section{Une lecture queer}

\section{Aramesh Yaghoubi}

Résumé :

L'article propose une étude de la pièce 4.48 Psychose de Sarah Kane dans une perspective queer. Le texte invente un langage de l'ambivalence : un langage qui transgresse les catégories binaires. Le corps de la comédienne s'installe dans un espace mental et symbolique où plusieurs voix s'enchevêtrent et s'hybrident; cet espace est ambivalent, entre la vie et la mort, entre le sublime et l'obscène. La liminalité ici devient un espacetemps propice à la réappropriation du corps par une écriture qui se substitue à la chair.

This study of Sarah Kane's play 4.48 Psychosis puts forth in a queer perspective. The play sees the emergence, on stage and in text, of what Judith Butler refers to as "queer identity". Its text invents a language of ambivalence between voice and writing, between the masculine and the feminine: a language that transgresses binary categories. The body of the protagonist settles in a mental and symbolic space where several voices intertwine and hybridize; this space is ambivalent, between life and death, between the sublime and the obscene. The phase of liminality in which the piece unfolds becomes a space-time conducive to the reappropriation of the body by a writing that replaces the flesh.

La pièce 4.48 Psychose de Sarah Kane, écrite en 1999, mais présentée pour la première fois une année et demie après le suicide de la dramaturge, met en scène un espace mental où les voix anonymes font part de leur impossibilité de vivre ainsi que de leur résolution à mourir. Composée d'une série d'instants qui retracent l'expérience intérieure d'une femme prise dans un espace oscillatoire, la pièce alterne monologues intérieurs et dialogues imaginaires avec l'alter ego de Kane.

Dans cet article, il s'agira d'examiner les enjeux de l'ambivalence, qui est une dimension essentielle de la subversion de l'identité chez le sujet féminin dans la pensée et l'écriture de Kane. Comme la réflexion aboutit à la notion de subversion, la perspective queer sera favorisée avant toute autre. Ceci, parce que lequeer, dans son fondement, est construit sur une ambivalence, voire une polyvalence, des identités genrées.

L'ambivalence se manifeste dans la pièce de Kane par la remise en cause des catégories binaires du « masculin » et du « féminin ». Se pose la question suivante : dans quelle mesure 4.48 Psychose déstabilise-telle les catégories du genre et subvertit l'identité genrée du sujet?

Cette subversion du genre est suggérée dès le début de la pièce par le discours du sujet d'énonciation : «l'hermaphrodite brisée qui ne se fiait qu'à ille-même » (Kane, 2001: 10). Le mot ille est une création verbale 
rendant compte de l'ambivalence masculin / féminin, à l'encontre des langues qui séparent il et elle, lesquelles obligent le sujet à se définir et à se limiter; le langage de Kane transgresse cette limite, reformulant poétiquement les catégories binaires du genre. L'expression « l'hermaphrodite brisée » renvoie à la fêlure sexuée du sujet d'énonciation, matérialisé dans la traduction française par le trait d'union entre «ille » et «même ». Cet hermaphrodisme linguistique est aussi générique, comme le remarque Marion Chénetier-Alev, dans son article intitulé « Subversion des formes dans 4.48 Psychose ou l'invention d'un objet dramatique problématique » 2005: 74).

La transgression des genres ici intervient conformément à ce que Judith Butler appelle « la performativité du genre » (2005 [1990]). Butler dénonce par là l'action performative du langage qui, à travers la notion de genre, incite l'individu à se conformer à un modèle « homme » ou « femme » par son comportement, son langage, ses attitudes, tout au long de sa vie : performer alors signifie à la fois faire semblant, accomplir et répéter au double sens de reproduction mimétique et d'exercice avant une représentation théâtrale). La pièce de Sarah Kane fait voler en éclats cette binarité artificielle, théâtrale, du genre, par les enjeux du langage et du corps. II s'agit dans cet essai d'analyser l'ambivalence de cette pièce à travers la performativité du langage et du corps; puis, de comparer l'identité queer et l'identité du sujet féminin dans la pièce.

Kane, dans 4.48 Psychose, fait entendre et fait voir l'intériorité d'une psyché malade. La voix monologuée apparaît d'emblée être celle d'une femme, mais progressivement, à travers les dialogues intérieurs, nous prenons conscience de la présence d'un médecin-thérapeute, l'alter ego de Kane, à qui elle s'adresse fréquemment. Cette voix masculine donne à entendre la subjectivité « masculine » de l'intérieur du sujet d'énonciation, à savoir l'Autre. Ce sujet divisé pourrait être une parcelle parmi d'autres subjectivités cohabitant à l'intérieur de lui.

Ainsi, l'ambivalence dans 4.48 Psychose se manifeste par la complexification des différentes oppositions structurales : féminin / masculin; extérieur / intérieur; Moi / Autre; parole / silence; lumière / obscurité; imaginaire / réel; corps / voix; visible / invisible; vie / mort. En effet, Kane semble chercher à transgresser la définition binaire des catégories en brouillant et en déconstruisant leurs frontières. Or, la théorie queer, comme le montre Judith Butler dans Trouble dans le genre (2005 [1990]), remet en question les catégories sexuelles en déstabilisant les dichotomies de genre. Butler affirme que l'identité sexuelle du sujet est basée sur la performativité du genre : une série d'actes corporels et discursifs. Elle emprunte le concept de performativité à John L. Austin pour critiquer l'illusion d'une psyché intérieure préexistante : « la répétition parodique du genre révèle l'illusion de l'identité comme une profondeur irréductible et une substance intérieure » (2005 [1990]: 273).

\section{Ambivalence verbale. Queer et performativité du langage}


Dans 4.48 Psychose, le corps textuel est plus présent que le corps corps réel féminin. Les blessures du corps charnel se reportent sur celui du texte, sa structure, sa syntaxe, ses mots, ses lettres, sa ponctuation. C'est surtout le corps de la langue qui est troué, torturé. Le texte prend en charge le traumatisme. La déconstruction de la langue et l'autodestruction du sujet ont lieu simultanément.

Au plan formel et sonore, la pièce est écrite comme un poème en prose, sans didascalie, et est composée de vingt-quatre fragments séparés et monologués, comprenant des appels, des cris, des morceaux de réplique, des blancs typographiques, des séries de mots et des chiffres. Dans 4.48 Psychose, contrairement aux autres pièces de la dramaturge, le corps-chair est moins visible, au profit du langage. Autrement dit, c'est la corporalité de la parole qui intéresse davantage l'auteure. Significativement, la disposition et la typographie des mots sur la page, qui sont le support de la tension, mettent en valeur des contrastes et des changements de rythme et de registre. Elles sont les signes qui font ressortir la polyphonie intérieure du sujet.

Les enjeux textuels entre le silence et la parole établissent l'ambivalence du drame. Les silences se présentent sous deux formes, didascalique et typographique, mais n'indiquent pas l'absence totale de la parole. D'une certaine manière, le silence textuel est la présence d'une parole muette et non la disparition totale de la parole. En effet, les silences ne sont pas les signes de non-parole mais ceux d'une absence d'oralisation. Au fur et à mesure qu'on avance vers la fin du drame, les mots désertent la page et les blancs typographiques augmentent. Sur scène se joue la disparition progressive, annoncée, de la comédienne, jusqu'au dernier fragment, la page finale n'offrant plus qu'un blanc.

À cet égard, on peut parler d'une interaction entre l'action et la stagnation, ou bien d'une absence dans la présence et d'une présence dans l'absence. II ne s'agit pas de mettre en avant la stabilité d'une absence absolue, mais bien de tracer ce mouvement existentiel qui creuse l'absence dans la présence. Autrement dit, en s'approchant progressivement de la fin du drame, les silences (didascaliques ou typographiques) font appel à l'absence physique et à la disparition corporelle. Le sujet est présent par son absence. Ainsi, sa valeur d'existence réside dans l'empreinte de son corps disparu. La mort est considérée comme une survivance, une nouvelle apparition de l'être. Cette apparition-disparition est liée à la persistance de la parole après la disparition de corps :

regardez-moi disparaître

regardez-moi

disparaître

regardez-moi 
regardez-moi

regardez

$[\ldots]$

S’il vous plaît levez le rideau (Kane : 55-56.)

En plus d'établir les ruptures entre les phrases et les mots, les blancs typographiques signalent l'intériorité morcelée du sujet. Ils instaurent une rupture d'énonciation entre l'énonciateur et l'oralisation, donnant l'impression que la voix est suspendue. Pour Chénetier-Alev, les blancs typographiques «suggèrent quant à eux l'amuïssement graduel de la voix, et le déchirement du sujet entre différentes instances de discours » (7677). Ils ne sont pas muets et immobiles, mais performants et parlants; ils confèrent au texte la forme d'un poème et donnent à voir les mots sur la page comme des îlots abandonnés afin d'évoquer à la fois leur importance et leur précarité. Les fragments des voix constellent la page et dessinent un poème.

Ainsi le « morcellement théâtral et humain » (Delvaux, 2012: §23), pour utiliser les termes de Martine Delvaux, se retrouve aussi sur le plan des genres littéraires : théâtre et poésie. Chénetier-Alev a bien souligné l'oscillation entre la poéticité et la théâtralité de la pièce :

4.48 Psychose oscille ainsi entre théâtre, poésie. [...] L'œuvre articule dialogue et soliloque; discordance et musicalité; esthétique de la fragmentation (du court-circuit) et lyrisme (épanchement) [...]. (74.)

De plus, comme le souligne Delvaux, la stratégie de la mise en abyme ou du théâtreintérieur dans le théâtre met en évidence la fragmentation-hybridation de l'imaginaire et du réel, ou du rêve et de la vie. En d'autres termes, l'expérimentation dramatique de Kane fait en sorte que le théâtral et le poétique se rejoignent, ne font qu'un. On peut réaffirmer l'hybridation dans cette réplique : «Rien qu'un mot sur une page et le théâtre est là » (Kane : 19). En effet, l'ambivalence des identités sexuées et celle du genre dramatique accroît le caractère subversif de la pièce.

\section{Ambivalence de l'oralité féminine. Entre silence et parole}

Alors que nous assistons à une disparition du corps du sujet parlant, la performativité émerge dans le corps de la parole. Le corps de chair est réduit à des voix hors-scène. La voix devient l'objet même du discours et, dans cette perspective, on peut parler de « théâtre des voix intérieures », pour reprendre l'expression de Jean-Louis Chrétien (2009: 205-210). Une seule voix polyvalente envahit l'espace. Ce monologue intérieur est dialogique et empreint d'ambivalence et d'hybridité. L'interaction des paroles monologuées se révèle être une poétique qui met au jour une cohabitation des contraires. L'ambivalence exerce une dialectique entre les entités 
opposées, un mélange paradoxal qui lie l'expression de la douleur de l'être à la recherche de l'idéal. De cette manière, l'auteure, en faisant dialoguer les monologues, établit une tension entre le Moi et l'Autre à l'intérieur de soi.

L'énonciation chorale, polyphonique, représente à la fois le caractère hétérogène et morcelé des paroles et constitue le dispositif idéal de l'ambivalence. La voix féminine et la voix masculine s'échangent les mots dans un « Moi » choral. La superposition de multiples voix fait en sorte que la parole s'enchevêtre et se disperse. Cette polyphonie évoque une sorte de choralité qui réfère à la cohabitation de différentes paroles. L'orchestration de la scène produit alors une rupture entre les unités de parole pour mettre en avant une intériorité morcelée et fragmentée qui témoigne de la vulnérabilité de la parole unique, donc de la fragilité d'un sujet unifié.

Cette parole performante devient une force qui permet à l'auteure de mieux se connaître et d'établir un lien entre le Moi et l'Autre. Elle est connaissance de soi. La voix n'est pas qu'intérieure, elle est aussi un dispositif liminaire entre le dedans et le dehors du corps. Cette performantivité, selon Chénetier-Alev, désigne « une oralité du cri silencieux » (72) ou ce que Kane nomme « une symphonie solo » (52). On comprend par cette expression que l'oralité paradoxale de l'œuvre tente de se présenter comme un drame muet. Ainsi, la choralité est un dispositif qui révèle à la fois les caractères homogène et hétérogène de la voix énonciative. Ces voix réflexives engendrent finalement une situation qui mènerait à la dépersonnalisation du sujet ou à une sorte de « désidentité » (Grossman, 2008: 12).

La répétition est une modalité particulière du rythme, conférant une sonorité, une raie de résonances à la langue. Dans le texte de Kane, elle fait naître le caractère poétique de l'écriture :

Brille scintille cingle brûle tords serre effleure cingle brille scintille cogne brûle flotte scintille effleure scintille cogne scintille brille brûle effleure serre tords serre cogne scintille flotte brûle brille scintille brûle Kane, 39)

Scintille cogne cingle effleure tords serre brûle cingle serre cingle cogne scintille brille serre brûle cogne effleure scintille flotte brille scintille effleure serre brûle cingle serre cingle cogne brille scintille brûle (Kane, 41)

Le langage selon Butler est lui-même le lieu d'une répétition, d'une citation constante et, conséquemment, il ouvre la porte à l'agentivité du sujet à travers le redéploiement, la resignification et la répétition subversive :

Si les normes et les discours nous constituent au travers de ce qu'ils nous disent et nous empêchent de dire, ils nous donnent également une «puissance d'agir » (Butler, 2004 [1996]), [...] parce que ce 
caractère performatif du langage, qui fait advenir ce dont il parle, procède d'une dimension rituelle qui suppose la répétition du discours. (Allard-Poesi et Huault, 2012: 48.)

Le langage poétique, lui aussi lieu de la répétition, devient celui de la construction du sujet : il trouve dans le langage les ressources de son agentivité pour transformer une situation qui l'opprime. Julia Kristeva dans $L a$ révolution du langage poétique (1974), estime que le langage poétique nous donne accès à ces pulsions dans leur multiplicité fondamentale : il existerait un rapport de causalité nécessaire entre l'hétérogénéité des pulsions et les possibilités plurivoques de la poésie.

La poétique de la répétition dans 4.48 Psychose révèle le pouvoir de l'esthétique chez Kane et une autre manière de concevoir la réalité. Selon Kristeva, «le langage poétique, étant donné sa capacité à perturber, à subvertir et à déstabiliser l'exercice de la loi paternelle [le symbolique], permet de recouvrer le corps maternel dans la langue » (Kristeva parahrasée par Butler, 2005 [1990]: 180). Par conséquent, le langage poétique est une source de subversion. Ainsi, la répétition des mots dans 4.48 Psychose n'a rien d'un hasard. Elle fait partie intégrante de la performativité, elle constitue le moyen le plus immédiat d'inscrire le rythme à même la structure de la pièce.

Si l'écriture et de la parole chez Kane peuvent être analysées comme des matériaux qui mettent en œuvre l'ambivalence pour fusionner la textualité et l'oralité de la pièce, comment l'ambivalence surgit-elle du corps ou de l'imaginaire du corps?

\section{Ambivalence du corps. Queer et performativité du corps}

Contrairement aux autres pièces de Kane, 4.48 Psychose confronte le spectateur à un corps physiquement immobile sur scène, réduit à sa seule fonction vocale. Dans ce théâtre mental, l'émergence du corps est fantasmée. L'image du corps unique semble disparaître et le corps ne cesse d'apparaître et de disparaître dans un mouvement oscillatoire conduit par la voix : "Le corps s'absente alors pour ne plus laisser "voir" que la voix, [...] la voix, ou ce qu'il reste du corps. » (Angel-Perez, 2011: 29.) Effectivement, la polyphonie des voix étrangères conduit à une spectralisation du corps. Une telle prise en charge du corps produit un «paradoxe phénoménologique », selon l'expression d'Elisabeth Angel-Perez 2013). Autrement dit : « moins on voit le corps plus on le voit et plus on expose le corps, moins on le voit) » Angel-Perez, 2013).

Mais l'effacement, l'absence ou l'immobilité du corps réel ne sont pas les signes de sa futilité. À plusieurs reprises dans le texte, l'existence corps charnel s'affirme : «Vous croyez qu'il est possible de naître dans le mauvais corps? » (Kane : 21); “C'est moi-même que je n'ai jamais rencontrée, dont le visage est scotché au verso de mon esprit » (55); « Je suis grosse » (11); « Mes hanches sont trop fortes / J'ai horreur de mes organes génitaux » (12). 
Au-delà de la division verbale du sujet, l'image du corps réel unique se révèle aussi divisée et fragmentée. La transgression que nous avons observée dans le langage et les voix glisse vers le corps. Au cours de la pièce, le sujet s'adresse régulièrement à un homme, le thérapeute, un double ou un alter ego, comme une figure du Moi idéal du sujet. Plus tard, il exprime son désir d'avoir une amante : « une femme me manque qui n'est jamais née / j'embrasse une femme par-delà les ans qui disent que jamais on ne se rencontrera » (24); «Une chanson pour mon aimée, touchant à son absence » (25). Ces dialogues intérieurs tracent une identité corporelle potentiellement diverse, multiple et hybride. On peut imaginer un sujet à la fois homosexuel, bisexuel, hétérosexuel, transsexuel, ou même asexué quand il dit : « Voyez l'Eunuque1 de la pensée châtrée » (52), « Je ne peux pas baiser » (11). Cette plasticité des figures féminine et masculine construit l'identité subversive d'un seul sujet d'énonciation, en donnant chair et voix à un Autre méconnu du sujet.

La représentation du corps dans 4.48 Psychose implique une transgression scénique, mais aussi une transgression du genre. La performativité des catégories genrées se joue sur les frontières du corps, les frontières étranges du corps. Cette « inquiétante étrangeté » (Freud, 1988 [1985]) est propre à la présence « inhumaine » de l'Autre (Butler, 2005). Cette zone floue, hybride et poreuse produit une tension dans la pièce et met en œuvre une performativité à l'instar des identités postmodernes queer. Les identités sexuées, qui déstabilisent les normes du genre, apparaissent indécidables.

Butler explique également la performativité comme une modalité qui permet de pratiquer le genre. La performativité permet au genre d'être « en quelque sorte un "acte" » (Butler, 2005 [1990]: 273) et à « l'identité [d'être] comme un effet [...] [qui] ouvre des possibilités en ce qui concerne la "capacité d'agir” » (273), et le genre est une « répétition stylisée d'actes » (265). Butler montre que le corps est ambivalent entre le sexe et le genre. La pensée féministe postmoderne ébranle l'idée que « le sexe » serait une donnée naturelle et montre que le corps est un concept historique. Pour Butler, le masculin ou le féminin n'existent pas préalablement, ce sont l'énonciation et la répétition des genres normatifs qui leur permettent d'exister (Butler, 2005 [1990]: 10). Ainsi, il n'y a pas d'identité genrée préexistante.

Les identités sexuelles peuvent être facilement vulnérables, oscillant entre les diverses catégories hommefemme : lesbienne, gai, bisexuel, drag, butch-fem, transsexuel, transgenre, etc. La théorie butlerienne de la performativité remet ainsi en question les préjugés sur les identités basées sur la stabilité du genre et du sexe. Elle peut aider à reconnaître le féminin en dehors de la normativité du genre.

Le théâtre de Kane, comme celui du queer, se fonde sur la vulnérabilité du sujet à travers la transgression des unités verbales et non-verbales. À l'instar de l'avènement de l'ambiguïté des genres féminins et masculins qui peut faire peur (Butler, 2012), Kane, en voulant devenir une subjectivité singulière, transforme la négativité de l'existence en vitalité de l'écrit et de la parole. L'auteure accepte de prendre le risque de cette angoisse pour 
fréquenter les frontières de ce qu'Évelyne Grossman nomme l'« inhumain » (2010). Cette angoisse est celle de l'expérience d'une déshumanisation — une « expérience-limite » (Blanchot, 1969: 67) — au profit d'une subjectivité non-personnelle, que Gilles Deleuze et Félix Guattari nomment «le devenir animal » (1975: 24).

Cette angoisse de la mobilité genrée permet au sujet de se dissocier de soi dans l'acte limite de l'écriture afin de se décoller de l'être. En effet, l'ambivalence prépare le corps pour la transgression qui est un dépassement des limites de soi) et ouvre la porte à sa déconstruction. L'esthétique de l'ambivalence est basée sur la tension et le trouble dans les catégories binaires. Cette déstabilisation fait du théâtre de Kane une esthétique de l'entre-deux, une dramaturgie de la brèche ou un théâtre liminaire : « Je chante sans espoir sur la frontière » (Kane: 19). La « liminalité2 » est attribuée aux entités dichotomiques (homme et femme, extérieur et intérieur, imaginaire et réel, visible et invisible, vie et mort, etc.) qui surgissent par le corps. Or, la cruauté du corps décomposé et morcelé, produite par une liminalité du dehors et du dedans, de l'humain et de l'inhumain, est la source de cette inquiétante étrangeté au sein du théâtre kanien.

La souffrance, chez Sarah Kane, est basée sur la volonté d'une « sortie de soi » (Grossman, 2008: 12) qui est aussi une perte de soi, comme le « hors de soi ». La décomposition du sujet, quand il se dédouble, témoigne de sa mort. Dès qu'il est dans l'écriture, il joue avec les mots et commence à mourir. Le sujet est hanté par une multitude de voix intérieures $\underline{3}$ qui parlent en lui, le troublent et le défigurent (Grossman, 2004). Ces voix glissent dans l'écriture et deviennent une façon d'entretenir l'identité. D'une certaine manière, l'énonciation est comme une réappropriation, et donc une double appartenance du corps.

\section{Liminalité mort-vie ou ambivalence sublime-obscène}

L'autre forme de l'ambivalence dans 4.48 Psychose est la transgression du clivage entre la vie et la mort. Toutes deux cohabitent sur la frontière, tout en demeurant opposées. Le protagoniste franchit sous nos yeux une frontière depuis toujours associée aux limites de la vie. La pièce montre ainsi la voie vers un nouveau possible et réorganise les rapports qu'entretient l'humain avec lui-même et le monde. Ici, l'expression du désir de vivre est étroitement liée au désir de mourir. Ces deux tensions se côtoient, se complètent, malgré leur divergence, et forment une conciliation, une hybridation.

La transition de la vie à la mort devient la matière théâtrale par excellence. Cette phase de l'entre-deux convoque la violence et l'horreur, mais, au-delà de cela, elle réalise un contact avec le sublime. Autrement dit, la souffrance et la violence du sujet kanien envers soi-même créent une lucarne vers le sublime ou, pour reprendre les termes de Delvaux, « une lumière au bout du tunnel » (\$26). La luminosité revient à plusieurs reprises dans le texte et fait écho à la rencontre de l'inconnu ou de l'invisible, voire de l'impossible. C'est pourquoi la maladie, le chaos, la douleur, l'aliénation psychique, bref, tout ce « qui rend abject » (Kristeva, 
1980: 13), ne sont qu'un moyen d'atteindre l'extase et l'amour immortel et le sublime. Cet amour inaccessible et sollicité au cours de la pièce n'est qu'une soif de l'inaccessible sacré; or, cela n'est possible qu'à travers l'obscène : « ce n'est [...] pas l'absence de propreté ou de santé qui rend abject, mais ce qui perturbe une identité, un système, un ordre. Ce qui ne respecte pas les limites, les places, les règles. L'entre-deux, l'ambigu, le mixte. 》 (Kristeva, 1980: 13.)

Le personnage de 4.48 Psychose parle en ce sens : «Belle douleur qui dit que j'existe » (41); «Rappelez-vous la lumière et croyez la lumière. Rien n'est plus important. » (38.) Et cette lumière ne réside que dans l'amour pour la mort. La souffrance semble donc être une voie incontournable pour accéder à l'émancipation, à la vérité et à l'idéal :

\author{
Coupez-moi la langue \\ arrachez-moi les cheveux \\ extirpez-moi les reins \\ mais laissez-moi mon amour \\ je préférerais avoir perdu mes jambes \\ m'être fait sauter les dents \\ m'être fait gicler les yeux \\ qu'avoir perdu mon amour (39)
}

Le personnage kanien, pour qui l'amour est la seule vérité, choisit la mort comme mode d'accès à une autre vie idéale. Le point culminant de la lucidité du sujet kanien réside dans sa conscience par rapport à l'amour, à la lumière, à Dieu ou à la vérité. Et « c'est dans ce rêve d'amour que se manifeste la survivance » (Delvaux: §28). L'ambivalence scénique et la mort par la violence sont comme « l'exigence d'entrer dans la danse », suggère Delvaux (§32), « la mort [qui] arrive comme le prix à payer pour être aimée [...]. C'est ainsi [que Kane] survit, dans cette quête d'amour » (Delvaux: §27-28).

Une telle émancipation ou rédemption du sujet ne se réalise que dans l'acte violent de la transgression des limites. Claude Régy le confirme dans un entretien avec David Gwénola : « [L]'effondrement des frontières entre des éléments officiellement opposés est un acte violemment subversif. 》 (Régy cité par Gwénola, 2002: n. p.) Régy ajoute :

Elle se situe dans un entre-deux, entre le non-désir de vivre et le non-désir de mourir, entre l'homme et la femme. L'hermaphrodisme fuse d'ailleurs de toutes ses œuvres. Elle est dans l'indéfini de son identité sexuelle, dans l'indéterminé de son désir, au milieu de ce vide. Et elle nous parle depuis cette sphère inexplorée qui frôle des états identifiables sans jamais s'y réduire. Dans cette cavité inconnue, tout 
résonne avec un sens nouveau. (n. p.)

Or, la peur de l'altérité est au cœur des frontières de cet état-limite. L'effondrement des limites d'une personnalité ouvre un espace vide et menaçant pour l'Autre. Le Moi se trouve dans l'entre-deux du dehors et du dedans, dans le flou entre le féminin et le masculin, entre l'imaginaire et le réel, la vie et la mort. Pourtant, cette vulnérabilité offre un champ de possibilités inédites à la reconnaissance de soi. Butler affirme d'ailleurs que :

Notre conscience se trouve perdue, perdue dans l'Autre, elle devient extérieure à elle-même, Autre à elle-même ou même dans l'Autre. La reconnaissance commence donc avec l'idée que l'on est perdu dans l'autre, absorbé dans et par une altérité que l'on est et que l'on n'est pas. La reconnaissance est motivée par le désir de se trouver réfléchi [...]. (2012: 272.)

\section{La subversion féminine et l'identité queer}

À la suite de ces observations, que devient l'identité du sujet féminin de la pièce? Cette œuvre de Sarah Kane peut-elle être considérée féministe? La dramaturge refusait d'assumer cette étiquette. Muriel Plana mentionne à cet égard que Kane n'est pas une auteure « du féminin ou de la féminité » (2012: 234.) Néanmoins, par sa manière d'illustrer la complexité des luttes et des dynamiques de pouvoir entre les personnages masculins et féminins - le personnage féminin matérialisant le désir de devenir « sujet » de son existence en choisissant une identité mixte, au confluent du genre et de l'héritage féminin familial —, l'œuvre apparaît féministe. Comme l'affirme Plana : «Pour une fois, une œuvre dramatique s'attache non aux vraies femmes (à l'Autre des hommes et donc aux hommes) mais aux femmes réelles, pour qui le masculin est à la fois Soi et l'Autre de Soi. » (234.)

Plana confirme aussi que « la seule ambition [du "théâtre des femmes" est] [...] d'échapper aux dualismes, aux stéréotypes et aux effets de mode » (236). Selon Mélanie Carpentier,

le postdramatisme - qui intègre de nouvelles normes comme « le fragment, la voix, la choralité, letrash, le monologisme, les écritures contemporaines... » (Plana, 2012: 239) — trop occupé à vouloir balayer les normes dramatiques, n'évolue plus et ne suit plus le mouvement des identités (celle du « féminin » notamment), qui, elles, ne cessent de bouger. (Carpentier, 2014: 110.)

La subjectivité féminine, nécessairement ambivalente, adhère à des idées hétérogènes. L'identité ambivalente constitue ainsi l'une des articulations d'une pensée hybride, riche, complexe, fluide et porteuse d'une polysémie immanente. Elle est constamment en hybridité, en division et dans la contradiction, dans le décalage et l'alternance. 
L'identité ambivalente est une trajectoire qui n'a de cesse de se faire et de se défaire. Elle est une oscillation permanente, s'opposant donc à ce qui est identifié comme totalement fixe ou stabilisé. La flexibilité des frontières des unités par leur transgression traduit l'ouverture d'une sexualité et d'un genre hybride. De ce fait, en prenant en compte la théorie queer, on comprend que le féminisme postmoderne n'est pas un féminisme identitaire. II déjoue la normativité, mais ne cherche pas à définir, à fixer l'identité. Donc, l'identité du sujet kanien reste performative, subversive et indécidable. Si nous nous appuyons sur l'ambivalence à l'instar du queer, c'est que l'identité du sujet queer est toujours précaire et subversive, bref en devenir.

\section{Conclusion}

L'esthétique de l'ambivalence dans 4.48 Psychose réside dans son caractère hybride et divisé à la fois. Autrement dit, ce que désire vraiment l'auteure n'est ni une identité hybride ni une identité divisée, mais une transgression des catégories traditionnellement présentées comme naturelles. Kane s'attache à dépeindre comment les principes binaires divisent profondément et violemment le sujet, ce qui amène à s'interroger sur le fondement de l'identité du sujet kanien.

L'action, pour ce sujet, comme pour celui de Butler, est construite dans le langage, lieu de répétition, qui ouvre la porte à l'agentivité du sujet pour transformer les situations opprimantes. Dans ce but, Butler cherche à mettre l'accent sur la performativité langagière qui surgit du corps, donc de l'identité sexuée. Autrement dit, la décomposition du corps et du langage est opérée afin d'inventer des figures plurielles, celles d'une identité en mouvement. Le féminin, dans 4.48 Psychose, rejette toute forme de fixation : il est traversé par le caractère mobile des identités et par la réinvention du rapport de soi à l'Autre. On est donc face à la subversion identitaire inouïe et infinie du féminin.

Or, par cette caractéristique subversive du verbe et du corps, ou d'imaginaire du corps, le mouvementqueer se différencie du féminisme historique et matérialiste, qui cherche à définir le féminin et le sujet féminin. La transgression des limites chez Kane est un moyen de fragiliser les frontières de la binarité sexuelle et genrée afin de les confronter à une nouvelle expérience, un « devenir autre » (Allard-Poesi, 2012: 53). En ce sens, la transgression est un devenir. La théorie queer plonge le sujet et sa capacité d'action ainsi que la pratique politique féministe dans une instabilité, une mouvance et une contestation continuelles. II s'agit de l'achèvement de la conception d'une identité féminine unifiée.

Tout bien considéré, 4.48 Psychose de Sarah Kane est une mise en scène de soi; la dégradation du corps et sa réappropriation dans la chair de l'écriture. Celle-ci, en tant qu'acte de subversion du langage, devient le lieu de subsistance et de survivance de l'auteure, et le livre-œuvre publié posthumément devient celui de l'identité. Le corps du livre est une matérialité fragile, mais mobile, et devient une porte ouverte à l'infinitude du Moi. 
1. Homme castré pour être gardien de harem.

2. « Le terme de liminalité, qui est le moment crucial du rituel, est emprunté à la théorie de l'anthropologue Victor Turner. Elle se présente comme le champ de la pure possibilité. Elle est le trait qui signale une situation sociale prête à accueillir des retournements radicaux des structures présentes, où les composantes du système culturel avec ses symboles subissent une sorte de déconstruction-recomposition ludique opérée par les êtres liminaires, sujets qui refusent le principe d'identité, leur caractère primaire étant la métamorphose. Selon Turner, la performance est le moyen de réalisation de cette réécriture des codes culturels dans la zone grise de la liminalité : c'est à travers la mise en scène de son corps que l'être liminaire déstructure le système culturel et crée du nouveau à partir de ce qui est déjà connu. » (Patera, 2014.)

3. La multitude des voix sur scène correspond à la pluralité de l'imaginaire du sujet. L'imaginaire des écrivains postmodernistes est un reflet de la défiguration, celle du corps et du langage, pour inventer des figures plurielles d'une identité en mouvement.

\section{Bibliographie}

Allard-Poesi, Florence et Isabelle Huault. 2012. « Judith Butler et la subversion des normes. Pouvoir être un sujet », dans Olivier GeRmAIN (dir.), Les grands inspirateurs de la théorie des organisations, préface de Nils Brunsson. Paris : EMS, «Les Grands Auteurs », t. I, p. 45-62. <https://halshs.archivesouvertes.fr/halshs-00733006>.

Angel-Perez, Elisabeth et Alexandra Poulain. 2011. «Le corps (ou ce qu'il en reste) sur la scène anglaise contemporaine », dans Passions du corps dans les dramaturgies contemporaines Villeneuve-d'Ascq :

Presses Universitaires du Septentrion, «Arts du spectacle. Théâtre », p. 29-44.

Angel-Perez, Elisabeth. 2011. “Blasted (Anéantis)" de Sarah Kane et le théâtre post-traqique».

Comparatismes en Sorbonne, no 2 «Spectacles de la violence ». łttp://www.crlc.paris-

sorbonne.fr/pdf revue/revue2/Spectacle5.pdf $>$.

BLANCHOT, Maurice. 1969. L'Entretien infini. Paris : Gallimard, 640 p.

ButLER, Judith. 2003. Antigone. La parenté entre vie et mort, traduction Guy Le Gaufey. Paris : EPEL, 103 p.

ButLeR, Judith. 2004. Le Pouvoir des mots. Politique du performatif, préface de Charlotte Nordmann et Jérôme Vidal, traduction de Charlotte Nordmann avec la collaboration de Jérôme Vidal. Paris : Éditions Amsterdam, $256 \mathrm{p}$.

a

BUtLER, Judith. 2005. Humain, Inhumain. Le travail critique des normes. Entretiens traduit de l'anglais

(Etats-Unis) par Christine Vivier et Jérôme Vidal. Paris : Éditions Amsterdam, 154 p.

b

BUtLER, Judith. 2005 [1990]. Trouble dans le genre. Pour un féminisme de la subversion traduit par

Cynthia Kraus. Paris : La Découverte, 283 p.

ButLER, Judith. 2012. Défaire le genre, traduit par Maxime Cervulle. Paris : Éditions Amsterdam, 311 p.

Carpentier, Mélanie. 2014. «Postdramatisme et esthétique de l'indécidabilité dans "Cleansed” et

"Phaedra's Love" de Sarah Kane ». Mémoire de maîtrise. Littérature Comparée, Université de Montréal, 
121 f. $<$ https://papyrus.bib.umontreal.ca/xmlui/handle/1866/11647>.

ChÉnetIer-Alev, Marion. 2005. «La subversion des formes dans "4.48 Psychose”, ou l'invention d'un objet dramatique problématique ». L'Annuaire théâtral, no 38 « La Subversion dans les dramaturgies anglaises contemporaines ", automne, p. 68-86. <https://www.erudit.org/fr/revues/annuaire/2005-n38annuaire3684/041615ar.pdf >.

CHRÉTIEN, Jean-Louis. 2009. Conscience et Roman. I, La Conscience du grand jour. Paris : Éditions de Minuit, «Paradoxe », $287 \mathrm{p}$.

Deleuze, Gilles et Félix Guattari. 1975. Kafka, pour une littérature mineure. Paris : Minuit, 159 p.

Delvaux, Martine. 2012. «Mourir-Survivre. Lumière de Sarah Kane». Temps Zéro : Écritures Contemporaines. Poétiques, esthétiques, Imaginaires, vol. 5, no 5. $<$ http://tempszero.contemporain.info/document982>.

FreUd, Sigmund. 1985. L'inquiétante étrangeté et autres essais, traduit de l'allemand (Autriche) par Fernand Cambon. Paris : Gallimard, « Folio essais », 352 p.

Grossman, Evelyne. 2004. La Défiguration. Artaud-Beckett-Michaux. Paris : Minuit, 120 p.

Grossman, Evelyne. 2008. L'angoisse de penser. Paris : Minuit, 156 p.

Grossman, Evelyne. 2010. «Modernes déshumanités ». Alea, vol. 12, no 1, juin, p. 47-57. $<$ http://dx.doi.org/10.1590/S1517-106X2010000100004>.

GWÉNOLA, David et Claude RéGY. 2002. “4.48 Psychose”. Entretien avec Claude Régy ». Théâtre-

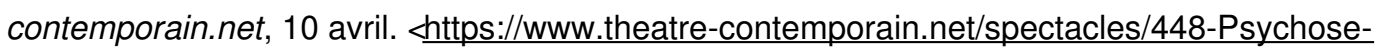
1334/ensavoirplus/idcontent/8611>.

KANE, Sarah. 2001. 4.48 Psychose, traduit par Evelyne Pieller. Paris : L'Archée, 56 p.

KrISTEVA, Julia. 1974. La révolution du langage poétique. Paris : Seuil, 645 p.

Kristeva, Julia. 1980. Pouvoirs de l'horreur. Essai sur l'abjection. Paris : Seuil, 256 p.

Patera, Teodoro. 2014. «Liminalité et performance. De l'anthropologie de Victor Turner aux "Folies Tristan" \#. Perspectives médiévales, no 35, mis en ligne le 1er janvier 2014. $<$ http://journals.openedition.org/peme/5025; DOI : 10.4000/peme.5025.

PlanA, Muriel. 2012. Théâtre et féminin. Identité, sexualité, politique. Dijon : Éditions Universitaires de Dijon, «Écritures », $363 \mathrm{p}$.

Angel-Perez, Elisabeth. 2013. «Éloge de l'ombre. Les paradoxes du corps spectral dans le théâtre anglais contemporain ». Miranda, no 8 « In Umbra Voluptatis », 28 juin 2013. . Consulté le 16 mai 2018. 